\title{
Resolución de hernia abdominal con pérdida de domicilio en dos tiempos quirúrgicos, en un paciente con obesidad mórbida
}

\author{
Resolution of abdominal hernia with loss of home in two surgical times, in a patient with \\ morbid obesity
}

\author{
Israel A. González-González, Daisy C. Zenteno-Martínez* y Francisco J. Campos-Pérez \\ Clínica Integral de Cirugía para la Obesidad y Enfermedades Metabólicas, Hospital General Dr. Rubén Leñero, Ciudad de México, México
}

\begin{abstract}
Resumen
La obesidad es un factor de riesgo para el desarrollo de hernias de la pared abdominal anterior. Las hernias incisionales se desarrollan hasta en el $13 \%$ de las laparotomías. Las más difíciles de reparar son las hernias recurrentes complejas y múltiples con pérdida significativa de domicilio. El mejor enfoque en el tratamiento de pacientes obesos y que concomitantemente tienen hernias de la pared abdominal anterior es aún un tema de debate. Presentamos el caso clínico de un paciente con obesidad mórbida y hernia abdominal con pérdida de domicilio, intervenido de cirugía bariatrica antes de la plastia ventral.
\end{abstract}

Palabras clave: Obesidad mórbida. Hernia incisional. Hernia con pérdida de domicilio. Cirugía bariátrica. Manga gástrica.

\begin{abstract}
Obesity is a risk factor for the development of anterior abdominal wall hernias. Incisional hernias develop in up to $13 \%$ of laparotomies: the most difficult to repair are complex and multiple recurrent hernias with significant loss of control. The best approach to treating obese patients who concomitantly have hernias of the anterior abdominal wall is still a matter of debate. We present a clinical case of a patient with morbid obesity and abdominal hernia with loss of residence, who underwent bariatric surgery before ventral plasty.
\end{abstract}

Key words: Morbid obesity. Incisional hernia. Hernia with loss of residence. Bariatric surgery. Gastric sleeve.

\section{Introducción}

La obesidad está asociada y también es causa de numerosas enfermedades importantes. Se considera uno de los factores de riesgo más comunes para el desarrollo de hernias de la pared abdominal anterior; esto puede explicarse por la presión intraabdominal elevada y la disminución de la distensibilidad de la pared abdominal por la obesidad'.

Las hernias incisionales se desarrollan hasta en el $13 \%$ de las incisiones de laparotomía: las más difíciles de reparar son las hernias recurrentes complejas y múltiples con pérdida significativa de domicilio (> 15$20 \%$ de los contenidos abdominales) $)^{2}$.

\section{Correspondencia:}

*Daisy C. Zenteno-Martínez

Jade 24 
Además del volumen de tejido graso subcutáneo e intraabdominal que presenta dificultades técnicas, la comorbilidad típica de los pacientes bariátricos, como la diabetes mellitus tipo 2, el síndrome de apnea obstructiva del sueño y la hipertensión arterial sistémica, supone un riesgo perioperatorio elevado ${ }^{3}$.

Actualmente, el mejor enfoque en el tratamiento de los pacientes que sean candidatos a la cirugía bariátrica o metabólica y concomitantemente tienen hernias de la pared abdominal anterior es aún un tema de debate debido a la ausencia de una base de evidencia para el consenso.

Una estrategia potencial es proporcionar un medio quirúrgico para corregir el peso del paciente antes de la reparación de la hernia ${ }^{4}$. Un concepto por etapas con un procedimiento de pérdida de peso inicial podría permitir la reposición de las vísceras herniadas, mejorar la comorbilidad y evitar el síndrome del compartimento abdominal en la reparación posterior ${ }^{3,4}$.

La cirugía bariátrica es el único tratamiento eficaz y sostenible de la obesidad mórbida y su comorbilidad a corto y largo plazo; la gastrectomía en manga laparoscópica se ha vuelto cada vez más popular debido a sus ventajas, que incluyen simplicidad técnica, un resultado restrictivo satisfactorio y baja incidencia o ausencia de complicaciones, como estenosis anastomótica y ulceración marginal, herniación interna, síntomas de dumping, deslizamiento y erosión, observadas en otros procedimientos bien establecidos.

La gastrectomía en manga laparoscópica es un procedimiento bariátrico seguro de primer paso que puede lograr una pérdida de peso satisfactoria y permitir a los pacientes de alto riesgo con obesidad mórbida someterse a una segunda operación no bariátrica ${ }^{5}$.

El neumoperitoneo progresivo preoperatorio (NPP) y la toxina botulínica tipo A son herramientas útiles en la preparación de los pacientes con hernias gigantes que han perdido el domicilio. Ambos son armas complementarias del procedimiento quirúrgico, especialmente con el uso de técnicas protésicas sin tensión, que permiten el manejo integral de estos pacientes ${ }^{6}$.

EI NPP es un procedimiento electivo en pacientes con hernias con pérdida de domicilio. Fue introducido en 1940 por Goñi Moreno, quien en 1947 publicó por primera vez su experiencia usando un neumoperitoneo inducido para el tratamiento de hernias grandes. Desde entonces, esta técnica ha sido modificada y utilizada en todo el mundo, con buenos resultados ${ }^{6,7}$.

Cuando la hernia de un paciente tiene un gran saco, que contiene grandes cantidades de vísceras, se producen cambios en el mesenterio, los intestinos, el tejido celular subcutáneo y la piel que envuelve el saco. Los retornos venosos y linfáticos se reducen debido a la compresión causada por el anillo fibrótico o defecto herniario en los bucles que sobresalen de la cavidad6.

Las hernias con pérdida de domicilio también causan contracciones progresivas de los músculos abdominales debido a la falta de vísceras, y reducen la presión y la capacidad de la cavidad abdominal. A medida que más intestinos abandonan el saco, la presión intraabdominal se reduce, disminuyendo el hemidiafragma y alterando el equilibrio ventilatorio, lo que causa restricción inspiratoria y espiratoria ${ }^{6,8-10}$.

El uso de NPP no se ha establecido ampliamente en la mayoría de los hospitales, aunque los grupos especializados que lo han incorporado han reportado buenos resultados con un riesgo aceptable.

Los objetivos que cumple son:

- Distensión progresiva de la pared abdominal para poder cerrar el defecto herniario con la menor tensión posible en el acto quirúrgico.

- Estabilizar la forma y la función diafragmática, mejorando la función ventilatoria.

- Elongación de los músculos de la pared abdominal y aumento del volumen de la cavidad abdominal.

- Disección neumática de bridas y adherencias viscerales, lo que facilita la reintroducción de las asas intestinales en la cavidad abdominal.

- Por último, se ha descrito irritación peritoneal y vasodilatación local reactiva, con aumento de los macrófagos los primeros días tras la aplicación, lo que mejora la capacidad cicatricial.

Es útil la realización de una tomografía computarizada (TC) abdominal, de ser posible en maniobra de Valsalva, para la evaluación de los diámetros y volúmenes del abdomen y del saco herniario, así como para valorar las características de la musculatura lateral y los rectos abdominales.

La definición radiológica de la hernia con pérdida de domicilio es una relación entre el volumen herniario y el volumen de la cavidad abdominal $>20 \%$, lo cual provocaría una reintroducción traumática a la cavidad.

La colocación del catéter para la insuflación de aire puede ser guiada por ecografía, que es lo más frecuente, o por TC, que proporciona un mejor control de la localización. Se insufla la cavidad con 200$300 \mathrm{ml}$ y se envía al paciente al departamento de radiología para confirmar el neumoperitoneo. Si la radiografía muestra aire libre en ambos hemidiafragmas, se completa la insuflación de aire; pueden 
introducirse volúmenes diarios de 1000-1500 ml, según la tolerancia del paciente. Un aspecto interesante es la monitorización de la presión intraabdominal durante la insuflación, que no debería exceder de $15 \mathrm{mmHg}$; en general, esta comprobación ha sido abandonada, ya que la experiencia indica que es muy difícil superar los $12 \mathrm{mmHg}$ sin que el paciente presente importantes molestias que hagan suspender la insuflación de aire. La administración diaria puede realizarse en visitas domiciliarias o en régimen de cirugía ambulatoria. Por último, al final del proceso se suele realizar un último control de TC abdominal, con el objeto de calcular el volumen de la cavidad y la nueva relación entre volumen herniario y volumen de la cavidad abdominal ${ }^{11}$.

No hay consenso en la literatura sobre la cantidad de aire que se debe insuflar en un programa de NPP, ni siquiera sobre cuánto tiempo debe mantenerse. Los informes sobre la duración del NPP oscilan entre 3 y 100 días.

Las complicaciones del neumoperitoneo son variadas. Se han reportado perforación intestinal y punción de órganos sólidos; el hematoma de la pared abdominal y el enfisema subcutáneo se informan comúnmente, y menos el neumotórax y el enfisema del mediastino y del pericardio $0^{7,12}$.

Por otro lado, la técnica de infiltración de toxina botulínica, a pesar de su relativamente reciente incorporación, es una herramienta útil en la preparación prequirúrgica de las hernias con pérdida de domicilio, al actuar como una separación de componentes «química" reversible y con mínimas molestias para el paciente. Además, presenta la ventaja de que la acción continúa en el posoperatorio tardío, unos 6 meses, en los que el abdomen operado va adaptando estos cambios ${ }^{11}$.

Aunque la reparación laparoscópica es un enfoque prometedor, no es aplicable a las hernias complejas con una significativa pérdida de domicilio $>15-20 \%$ de los contenidos abdominales). La preparación adecuada para la operación es obligatoria en los pacientes con grandes hernias incisionales ${ }^{2,11}$.

\section{Caso clínico}

Varón de 43 años con los siguientes antecedentes de importancia: apendicitis aguda con apendicectomía abierta hace 10 años; diverticulitis complicada hace 10 años que requirió laparotomía exploratoria con sigmoidectomía y colo-recto anastomosis término-terminal manual, que presentó dehiscencia de la anastomosis

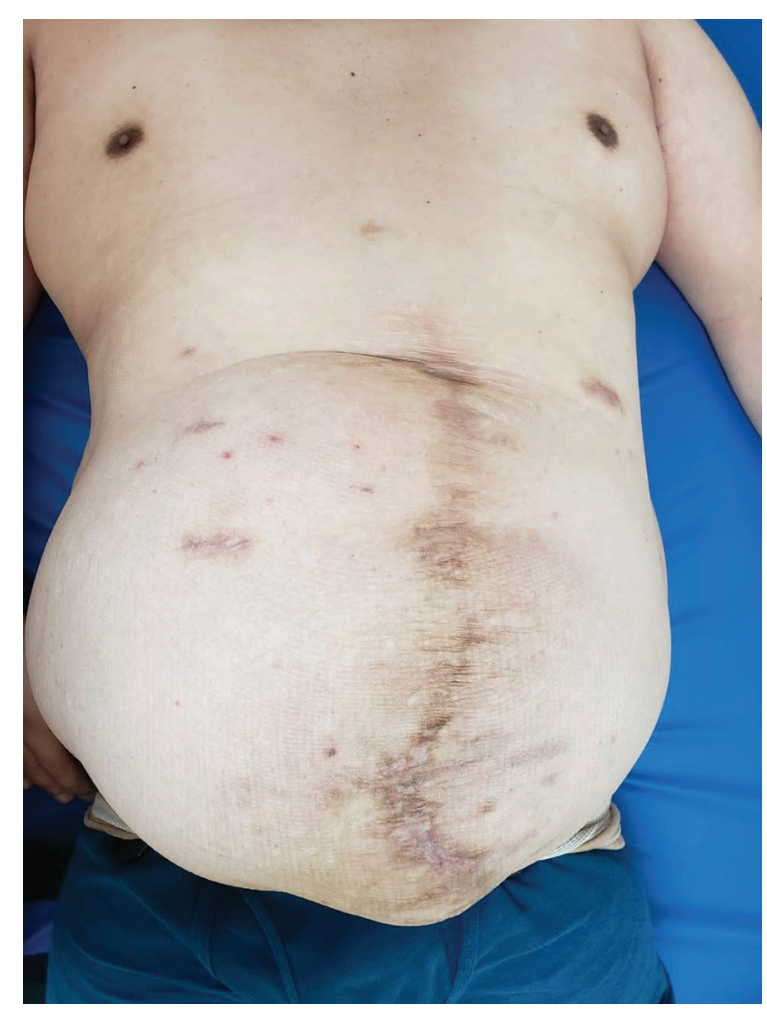

Figura 1. Visión anterior de la pared abdominal, con evidencia de hernia abdominal incisional con pérdida de domicilio.

y precisó colostomía durante un periodo de 9 meses, con restitución del tránsito intestinal hace 9 años; oclusión intestinal por adherencias hace 4 años, que requirió resección intestinal con anastomosis. Trastorno depresivo con ideación suicida hace 10 años, sin tratamiento. Alérgico a las cefalosporinas.

Acude por presentar una hernia ventral gigante con pérdida de domicilio de más de $30 \times 30 \mathrm{~cm}$, la cual le causa dolor y molestias de manera intermitente, desde hace 4 años. Presenta obesidad de grado III, con un índice de masa corporal (IMC) de $43.8 \mathrm{~kg} / \mathrm{m}^{2}$, desde hace 10 años. Posterior al cuadro de diverticulitis presentó ganancia de peso, y a pesar de realizar dieta y ejercicio no obtuvo resultados favorables, por lo que se le colocó un balón intragástrico por 9 meses, con pérdida de peso de $10 \mathrm{~kg}$ y reganancia de $12 \mathrm{~kg}$ posteriormente (IMC $44 \mathrm{~kg} / \mathrm{m}^{2}$ ) (Figs. 1 y 2).

Cuenta con TC abdominal, en la que se observan desplazamiento de asas, epiplón y estómago en el cuadrante superior (Fig. 3).

Debido a la pobre disminución de peso, se decidió realizar un plan terapéutico en dos tiempos quirúrgicos para la resolución de la hernia con pérdida de domicilio; en el primer tiempo se le realizó una manga 


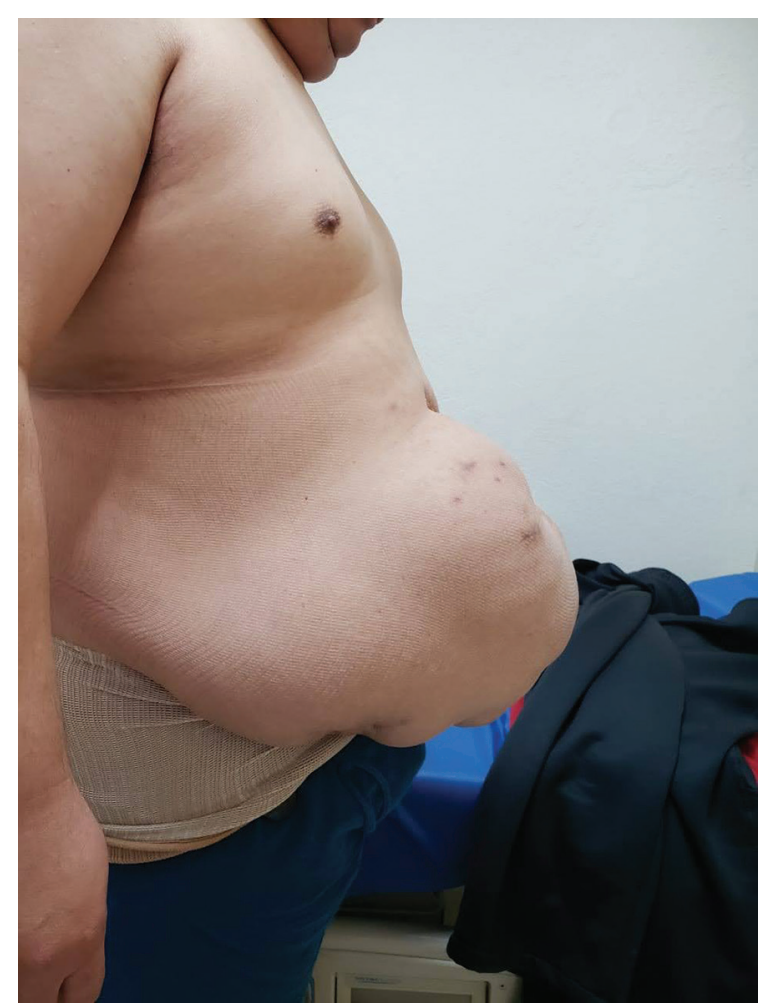

Figura 2. Visión lateral de la pared abdominal, con presencia de hernia con pérdida de domicilio.

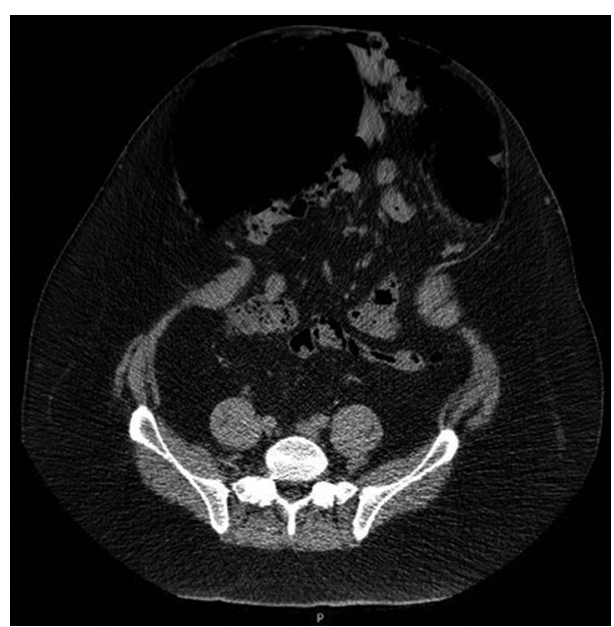

Figura 3. Tomografía computarizada en la que se observa hernia abdominal con pérdida de domicilio, gran cantidad de asas intestinales, epiplón y estómago en el cuadrante superior izquierdo.

gástrica laparoscópica, hace 1 año, con NPP durante 10 días con catéter hacia la cavidad e insuflación de aire ambiente con volúmenes de 1500-2000 ml, con recambios diarios, y toma de presión intraabdominal de $12 \mathrm{mmHg}$ (Fig. 4).

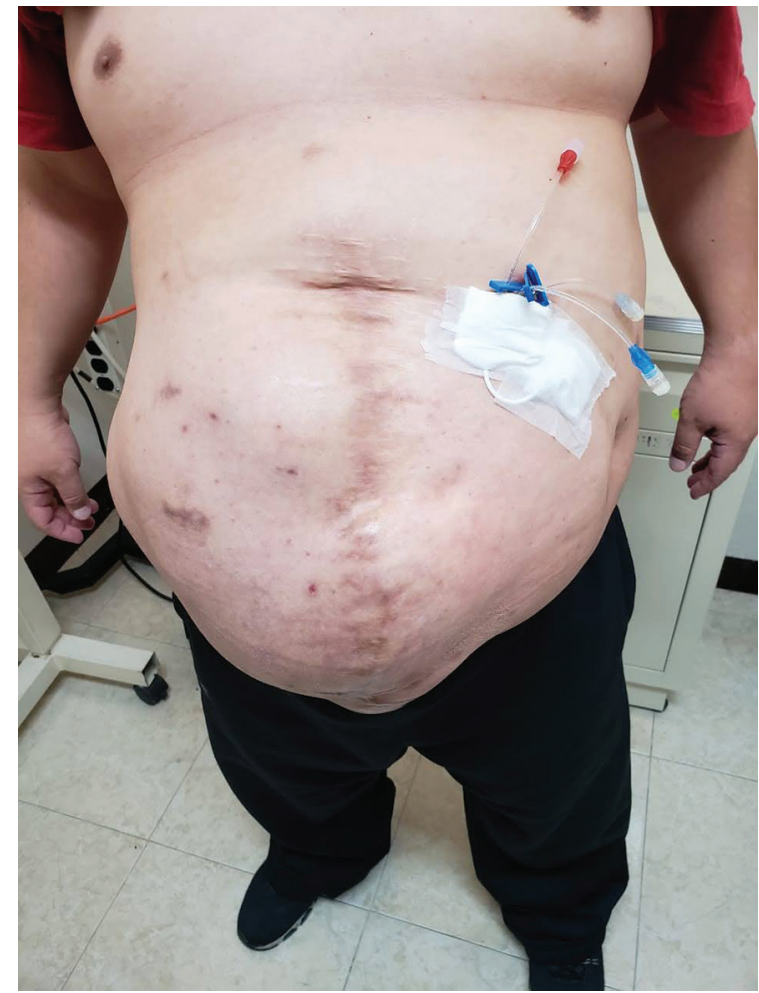

Figura 4. Catéter de insuflación para neumoperitoneo progresivo preoperatorio, colocado en el hipocondrio izquierdo evitando sitios de cicatrices previas.

A los 10 meses de realizar la manga gástrica tuvo disminución de peso con IMC de $37.6 \mathrm{~kg} / \mathrm{m}^{2}$, por lo que se decide un segundo tiempo quirúrgico en el cual se realizó plastia ventral con técnica TAR y colocación de seis mallas Progrip ${ }^{\circledR}$ de polipropileno con cierre isométrico, previa preparación con neumoperitoneo progresivo durante 15 días y aplicación de toxina botulínica (Fig. 5).

Se llevó a cabo la intervención quirúrgica y el paciente presentó una reacción anafiláctica grave a ciprofloxacino que ameritó manejo en terapia intensiva por 3 días.

Posteriormente presentó seroma e infección del sitio quirúrgico, por lo que se realizó un lavado quirúrgico con salvamento de mallas y se colocó un sistema de presión negativa por 3 semanas, con cierre de la herida de manera adecuada. El paciente fue egresado sin otras complicaciones a los 25 días de operado.

\section{Discusión}

La cirugía bariátrica, con sus ventajas de pérdida de peso y mejora de las comorbilidades, reduce significativamente las posibilidades de recurrencia en hernias no complicadas. Los buenos resultados 


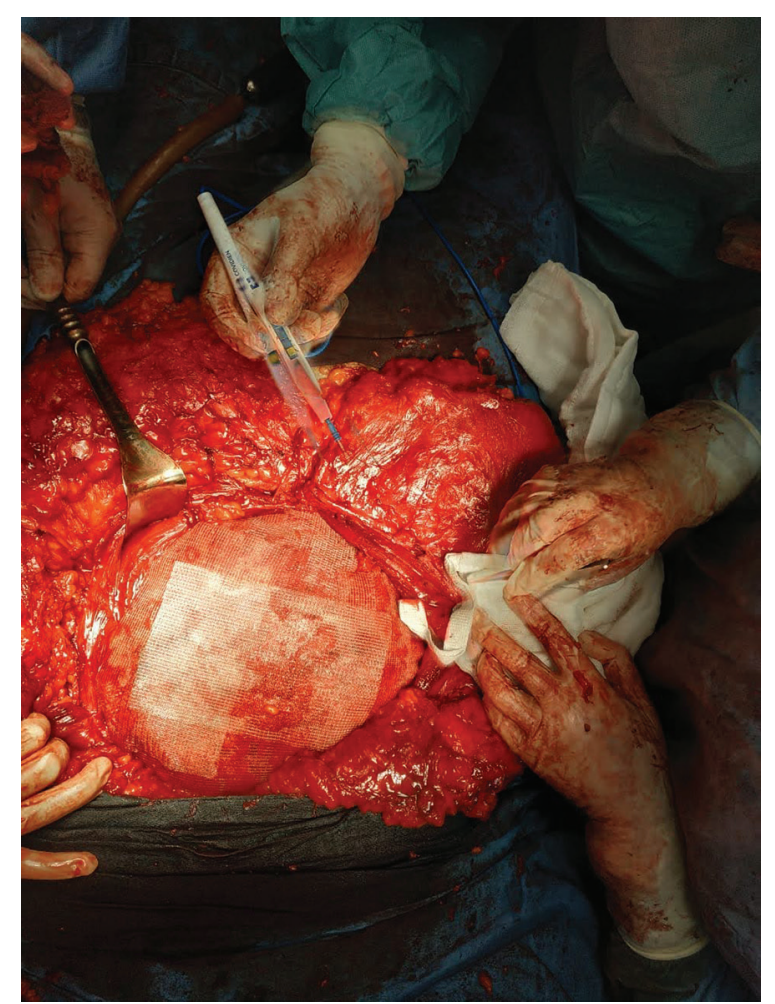

Figura 5. Colocación de seis mallas Progrip ${ }^{\circledR}$ para la reparación de la hernia con pérdida de domicilio.

muestran el camino a seguir en términos de seguridad al combinarlos. Por lo tanto, este enfoque debe considerarse seriamente en pacientes con obesidad mórbida que se presentan con hernia ventral primaria 0 recurrente. Sin embargo, esto solo debe ser realizado por equipos quirúrgicos con experiencia adecuada.

Las hernias de la pared abdominal con grandes sacos herniarios que retienen una gran cantidad de asas intestinales durante un largo tiempo son extremadamente difíciles de reparar. Se dice que estas hernias tienen "pérdida de domicilio» porque el contenido de la hernia excede la capacidad de la cavidad abdominal. La reducción forzada de estas hernias puede conducir a respiración insuficiente, síndrome compartimental abdominal e incluso incapacidad para completar la hernioplastia.

Para resolver este problema, la inducción de un neumoperitoneo progresivo se ha llevado a cabo preoperatoriamente durante más de 60 años. Según lo descrito por algunos autores, el mantenimiento del neumoperitoneo aumenta la capacidad de la cavidad abdominal retraída, realiza una lisis neumática de las adherencias intestinales y mejora la función diafragmática.

En general, un enfoque de dos pasos para tratar las hernias masivas con pérdida de domicilio en pacientes con obesidad mórbida es seguro y efectivo. La gastrectomía en manga como procedimiento de pérdida de peso inicial aborda la pérdida de domicilio con éxito, en algunas ocasiones sin necesidad de medidas preoperatorias adicionales para condicionar la reparación de la hernia y sin riesgo de síndrome compartimental abdominal. Este enfoque enriquece el arsenal de los cirujanos que se ocupan de la obesidad mórbida y las hernias complejas.

\section{Agradecimientos}

Agradecemos al equipo multidisciplinario de la Clínica Integral de Obesidad y Enfermedades Métabolicas del Hospital Dr. Rubén Leñero, Ciudad de México.

\section{Responsabilidades éticas}

Protección de personas y animales. Los autores declaran que para esta investigación no se han realizado experimentos en seres humanos ni en animales.

Confidencialidad de los datos. Los autores declaran que han seguido los protocolos de su centro de trabajo sobre la publicación de datos de pacientes.

Derecho a la privacidad y consentimiento informado. Los autores han obtenido el consentimiento informado de los pacientes y/o sujetos referidos en el artículo. Este documento obra en poder del autor de correspondencia.

\section{Financiamiento}

El presente reporte de caso no ha recibido ningún tipo de financiamiento.

\section{Conflicto de intereses}

Los autores declaran no tener ningún conflicto de intereses.

\section{Bibliografía}

1. Marzouk AMSM, Ali HOE. Laparoscopic ventral hernia repair combined with sleeve gastrectomy in morbidly obese patients: early outcomes. Surg J (NY). 2019;5:e87-e91.

2. Kingsnorth AN, Sivarajasingham N, Wong S, Butler M. Open mesh repair of incisional hernias with significant loss of domain. Ann R Coll Surg Engl. 2004;86:363-6.

3. Borbély Y, Zerkowski J, Altmeier J, Eschenburg A, Kröll D, Nett P. Complex hernias with loss of domain in morbidly obese patients: role of laparoscopic sleeve gastrectomy in a multi-step approach. Surg Obes Relat Dis. 2017; 13:768-73.

4. Newcomb WL, Polhill JL, Chen AY, Kuwada TS, Gersin KS, Getz SB, et al. Stage hernia repair preceded by gastric bypass for the treatment of morbidly obese patients with complex ventral hernias. Hernia. 2008;12:465-9. 
5. Hidalgo JE, Roy M, Ramirez A, Szomstein S, Rosenthal RJ. Laparoscopic sleeve gastrectomy: a first step for rapid weight loss in morbidly obese patients requiring a second non-bariatric procedure. Obes Surg. 2012;22:555-9.

6. Mayagoitia JC, Suárez D, Arenas JC, Díaz de León V. Preoperative progressive pneumoperitoneum in patients with abdominal-wall hernias. Hernia. 2006;10:213.

7. Elstner KE, Read JW, Rodríguez-Acevedo O, Ho-Shon K, Magnussen J, Ibrahim N. Preoperative progressive pneumoperitoneum complementing chemical component relaxation in complex ventral hernia repair. Surg Endosc. 2017;31:1914.

8. Parker SG, Halligan S, Blackburn S, Plumb AAO, Archer L, Mallett S, et al. What exactly is meant by "loss of domain" for ventral hernia? Systematic review of definitions. World J Surg. 2019;43:396.
9. López Sanclemente MC, Robres J, López Cano M, Barri J, Lozoya R, López S, et al. Progressive preoperative pneumoperitoneum in patients with giant hernias of the abdominal wall. Cir Esp. 2013;91:444-9.

10. Slater NJ, Montgomery A, Berrevoet F, Carbonell AM, Chang A Franklin $\mathrm{M}$, et al. Criteria for the definition of a complex abdominal wall hernia. Hernia. 2014;18:7-17.

11. Bueno-Lledó J, Torregrosa Gallud A, Jiménez Rosellón R, Carbonell Tatay F, García Pastor P, Bonafé Diana S, et al. Preoperative preparation of «loss of domain» hernia. Progressive pneumoperitoneum and botulinum toxin type A. Cir Esp. 2017;95:245-53.

12. Renard $Y$, Lardière-Deguelte $S$, de Mestier L, Appere $F$, Colosio $A$, Kianmanesh $\mathrm{R}$, et al. Management of large incisional hernias with loss of domain: a prospective series of patients prepared for progressive preoperative pneumoperitoneum. Surgery. 2016;160:426-35. 